\title{
Microglial p38 $\alpha$ MAPK is critical for LPS-induced neuron degeneration, through a mechanism involving TNF $\alpha$
}

\author{
Bin Xing ${ }^{1 \dagger}$, Adam D Bachstetter ${ }^{1 \dagger}$ and Linda J Van Eldik ${ }^{1,2^{*}}$
}

\begin{abstract}
Background: The p38 $\alpha$ MAPK isoform is a well-established therapeutic target in peripheral inflammatory diseases, but the importance of this kinase in pathological microglial activation and detrimental inflammation in CNS disorders is less well understood. To test the role of the p38 $\alpha$ MAPK isoform in microglia-dependent neuron damage, we used primary microglia from wild-type (WT) or p38 $\alpha$ MAPK conditional knockout (KO) mice in coculture with WT cortical neurons, and measured neuron damage after LPS insult.

Results: We found that neurons in co-culture with p38 $\alpha$-deficient microglia were protected against LPS-induced synaptic loss, neurite degeneration, and neuronal death. The involvement of the proinflammatory cytokine TNF $\alpha$ was demonstrated by the findings that $p 38 \alpha \mathrm{KO}$ microglia produced much less TNF $\alpha$ in response to LPS compared to WT microglia, that adding back TNF $\alpha$ to KO microglia/neuron co-cultures increased the LPS-induced neuron damage, and that neutralization of TNF $\alpha$ in WT microglia/neuron co-cultures prevented the neuron damage. These results using cell-selective, isoform-specific KO mice demonstrate that the p38 $\alpha$ MAPK isoform in microglia is a key mediator of LPS-induced neuronal and synaptic dysfunction. The findings also provide evidence that a major mechanism by which LPS activation of microglia p38 $\alpha$ MAPK signaling leads to neuron damage is through up-regulation of the proinflammatory cytokine TNF $\alpha$.

Conclusions: The data suggest that selective targeting of p38 $\alpha$ MAPK signaling should be explored as a potential therapeutic strategy for CNS disorders where overproduction of proinflammatory cytokines is implicated in disease progression.
\end{abstract}

Keywords: microglia, cytokines, knockout mice, p38alpha mitogen-activated protein kinase, neuron, tumor necrosis factor alpha

\section{Background}

Extensive evidence, both clinical and preclinical, implicates neuroinflammation and overproduction of proinflammatory cytokines as a contributor to pathophysiology of chronic neurodegenerative disorders such as Alzheimer's disease (AD), Parkinson's disease, and multiple sclerosis [for review, see: [1]]. Proinflammatory cytokine overproduction has also been documented as detrimental to recovery in acute brain injuries such as trauma or stroke [2-5]. In the brain, activated microglia are a major

\footnotetext{
* Correspondence: linda.vaneldik@uky.edu

+ Contributed equally

'Sanders-Brown Center on Aging, University of Kentucky, Lexington, KY 40536 USA

Full list of author information is available at the end of the article
}

mediator of neuroinflammation and can release a number of potentially neurotoxic substances, such as reactive oxygen species, nitric oxide, and various proinflammatory cytokines, of which two main proinflammatory cytokines TNF $\alpha$ and IL-1 $\beta$ are generally considered primary mediators leading to neurotoxicity [for detailed reviews on microglia, see: $[6,7]]$.

There are many critical roles for innate immunity, and thereby the primary effector cells, microglia, in the classically immune privileged CNS. For example, microglia are rapid responders to local tissue stressors [8,9], can efficiently clear apoptotic cells during neurodevelopment [10], and can promote neuro-repair through the production of growth factors [7]. The spectrum of activated

\section{() Biomed Central}


microglia phenotypes is diverse and generally beneficial. It is only when the activation becomes exaggerated or dysregulated does the response become neurotoxic. Therefore, it is of critical importance to elucidate the mechanisms that are specifically involved in the dysregulated response of microglia which contribute to neuronal damage.

Intracellular signal transduction cascades regulate the production of proinflammatory cytokines. By targeting a specific signal transduction pathway it is possible to determine if a pathway is involved in the dysregulated response that is neurotoxic and if the dysregulated response is amenable to intervention. One of the most well established signal transduction cascades that regulate the production of proinflammatory cytokines in peripheral tissue inflammatory diseases, such as rheumatoid arthritis, is the p38 mitogen activated protein kinase (MAPK) family $[11,12]$. The p38 MAPK family consists of at least four isoforms $(p 38 \alpha, \beta, \delta, \gamma)$, which are encoded by separate genes, expressed in different tissues and have distinct functions [13]. Activation of p38 MAPK signaling has been shown to regulate gene expression and lead to increased production of proinflammatory cytokines by a number of different mechanisms [for review, see: [14]]. The p38 MAPK pathway has been suggested to play a central role in various pathological CNS conditions including cerebral ischemia $[15,16]$ and Parkinson's disease [17-19], as well as in AD $[20,21]$, where postmortem studies find p38 MAPK activation occurs at the very early stage of the disease [20,22].

Previously we have shown using both a pharmacological approach with a selective small molecule p $38 \alpha$ MAPK inhibitor and a genetic approach with primary microglia that are deficient in $\mathrm{p} 38 \alpha$ that the $\alpha$ isoform of p38 MAPK is critical for the production of IL-1 $\beta$ and TNF $\alpha$ from activated microglia [23]. Moreover, suppression of $\mathrm{p} 38 \alpha$ MAPK with the small molecule inhibitor in an AD-relevant mouse model was also found to decrease brain proinflammatory cytokine production, and attenuate synaptic protein loss [24]. These data suggested that microglia $\mathrm{p} 38 \alpha$ MAPK is critical to inflammation-induced neurotoxicity. In the current study, we explored whether there is a causative link between microglia p38 $\alpha$ MAPK signaling and neuronal damage, as well as a potential mechanism for microglia-dependent neurotoxicity. We used primary microglia from either wild-type (WT) mice or from p38 $\alpha$ MAPK conditional knockout (KO) mice in co-culture with cortical neurons from WT mice. In WT microglia/neuron cocultures, LPS treatment led to a significant increase in TNF $\alpha$ production, loss of synaptic proteins, and neuronal death. Neurons in co-culture with p38 $\alpha$-deficient microglia showed reduced LPS-induced TNF $\alpha$ production and were protected against synaptic loss and neuronal death. The mechanism of neurotoxicity was explored by showing that addition of a neutralizing TNF $\alpha$ antibody prevented neuronal degeneration in WT microglia-neuron co-cultures, and addition of recombinant TNFa to KO microglia-neuron co-cultures led to enhanced neuronal degeneration. Our data support the conclusion that activation of p $38 \alpha$ MAPK and the downstream overproduction of the proinflammatory cytokine TNF $\alpha$ play a major role in the dysregulated microglial response to LPS that leads to neuron degeneration.

\section{Results}

Validation of microglia p38 $\alpha$ MAPK deletion in conditional $\mathrm{KO}$ mice

In the CNS, p38 $\alpha$ MAPK is not restricted to microglia; therefore, to determine the importance of $\mathrm{p} 38 \alpha$ MAPK specifically in microglia, we used primary microglia isolated from $\mathrm{p} 38 \alpha$ conditional $\mathrm{KO}$ mice, where $\mathrm{p} 38 \alpha$ is genetically deficient in microglia [25]. Microglia isolated from mice with the loxP-flanked p38 $\alpha$ allele but not carrying the Cre allele (p38 $\alpha$ WT) were found to have levels of $\mathrm{p} 38 \alpha$ MAPK similar to microglia from C57BL/ 6 mice (data not shown). We confirmed that this conditional gene deletion approach was highly efficient at eliminating the levels of $\mathrm{p} 38 \alpha$ MAPK from microglia as determined by immunoblotting. Specifically, microglia isolated from $\mathrm{p} 38 \alpha \mathrm{KO}$ mice showed essentially no p38 $\alpha$ compared to the p38 $\alpha$ WT microglia cells, either under control conditions or after treatment with LPS (Figure 1A). In addition, we confirmed the absence of p38 $\alpha$ in the microglia cultures from KO mice by immunocytochemistry (Figure 1B).

The absence of p38 $\alpha$ MAPK did not affect the number of microglia that were isolated from the p38 $\alpha \mathrm{KO}$ mice. It also did not affect the overall morphological appearance of the microglia in culture. For example, there was no obvious difference in the morphology of microglia in the p38 $\alpha \mathrm{KO}$ group compared to the p38 $\alpha$ WT group, as demonstrated by the microglia marker F4/80 (Figure 1B). These results were confirmed with two additional microglia-specific markers IBA1 and CD11b (data not shown). These data also documented that the microglia isolation method used resulted in a highly enriched population of microglia, with essentially $>99 \%$ purity for both the p38 $\alpha \mathrm{WT}$ and KO microglia cells.

\section{Microglial p38 $\alpha$ MAPK deficiency prevents LPS-induced neurotoxicity in microglia/neuron co-cultures}

Activated microglia are capable of secreting bioactive molecules, such as reactive oxygen and reactive nitrogen species, as well as proinflammatory cytokines, all of 


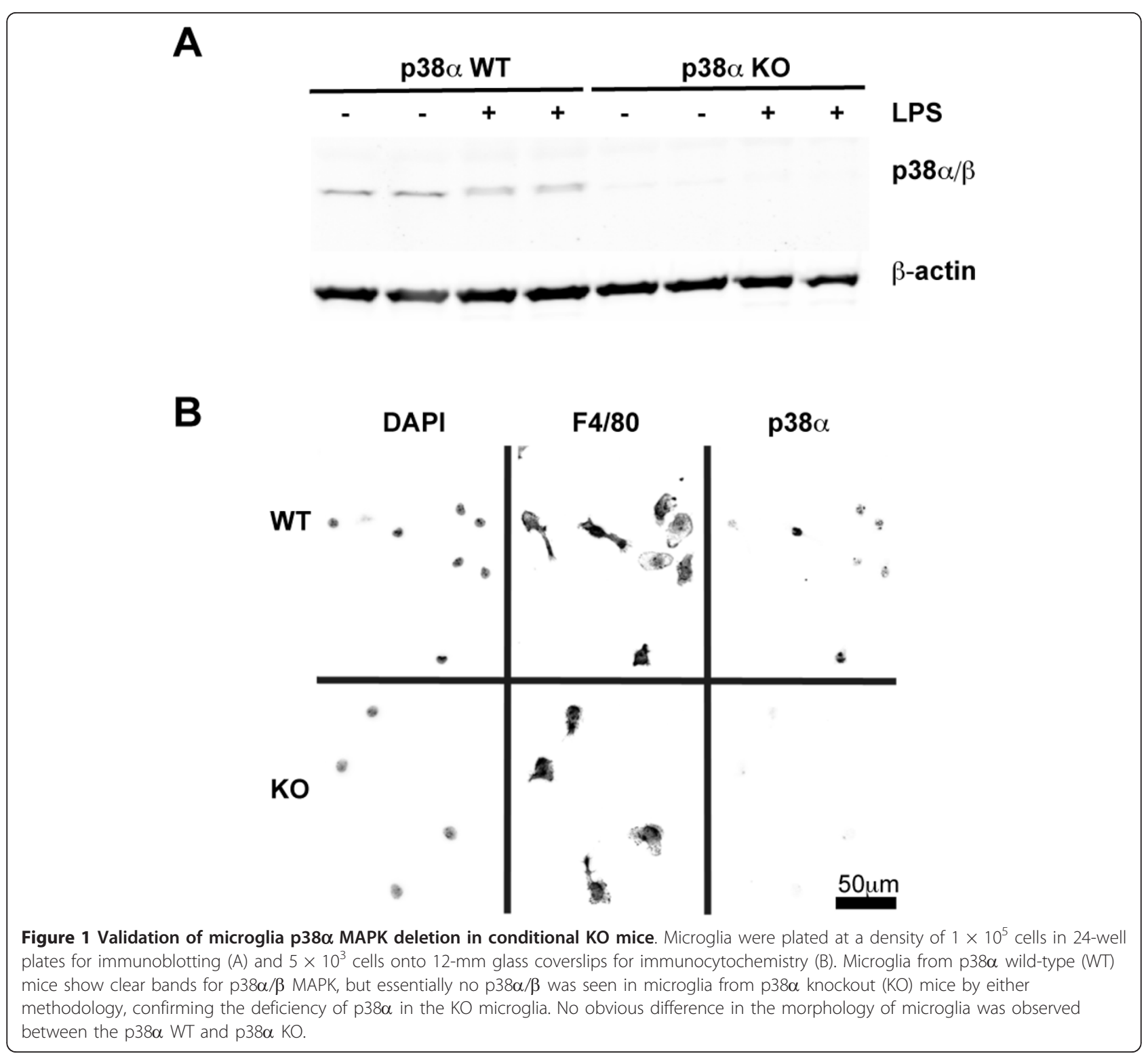

which have the potential to be neurotoxic [7]. We have previously implicated p38 $\alpha$ MAPK signaling as important for glia-induced neuronal death in a mixed glia/ neuron co-culture system [26]. However, p38 $\alpha$ MAPK is present in multiple CNS cell types, including microglia, astrocytes, and neurons. Therefore, in this study, we took a different approach to determine the specific contribution of microglia p38 $\alpha$ MAPK to glia-induced neuronal death. Specifically, we isolated microglia from either $\mathrm{p} 38 \alpha \mathrm{KO}$ or $\mathrm{p} 38 \alpha$ WT mice, placed them in coculture with WT primary cortical neurons, and tested whether the absence or presence of microglia p $38 \alpha$ would affect LPS-induced neurotoxicity. Consistent with what we previously reported [26], LPS had no effect on neuronal viability in the absence of microglia
$(100 \pm 1.3 ; 93.4 \pm 7.7 ; \%$ survival without and with LPS, respectively). Also as expected, treatment of WT microglia/neuron co-cultures with LPS for $72 \mathrm{~h}$ led to significant neuronal death, as determined by trypan blue exclusion assay (Figure 2). In contrast, WT neurons co-cultured with $\mathrm{p} 38 \alpha \mathrm{KO}$ microglia were resistant to LPS-induced neurotoxicity, showing essentially 100\% survival (Figure 2).

\section{Microglial p38 $\alpha$ MAPK deficiency attenuates LPS-induced} synaptic protein loss in microglia/neuron co-cultures We used the microglia/neuron co-culture system to address whether secreted factors from activated microglia can produce synaptic changes in the neurons and whether microglia p38 $\alpha$ plays a role in these responses. 
A

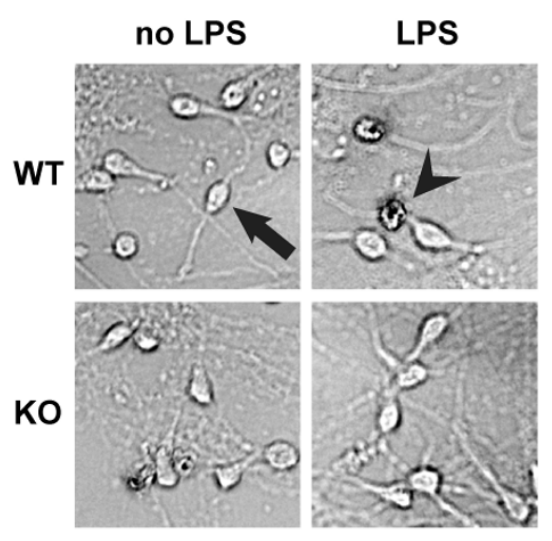

B

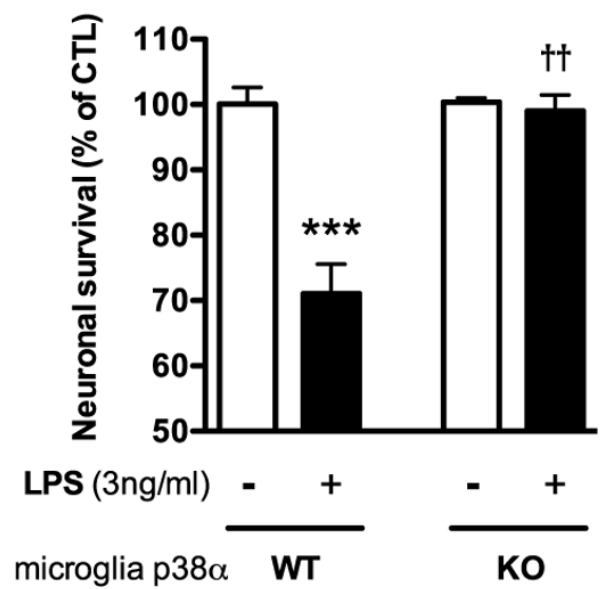

Figure 2 Microglial p38 $\alpha$ MAPK deficiency prevents LPS-induced neurotoxicity in microglia/neuron co-cultures. Primary cortical neurons were co-cultured with primary microglia from p38 $\alpha$ WT or p38 $\alpha$ KO mice. The microglia/neuron co-cultures were stimulated with 3 ng/ml LPS for $72 \mathrm{~h}$, then neurons analyzed by trypan blue exclusion assay. Representative photomicrographs of the neuron cultures are shown in (A). The arrow indicates a live neuron typical of the non-LPS stimulated co-culture. The arrowhead shows a dead neuron that is positive for trypan blue. In microglia from p38 $\alpha$ WT mice, LPS produced a significant decrease in neuronal survival $\left(B_{;}{ }^{* * *} p<0.001\right)$. No significant neuron death was seen in microglia from p38 $\alpha$ KO mice stimulated with LPS. Data represents 3 independent experiments.

We measured protein levels by immunoblotting (Figure 3A) for a panel of five synaptic proteins: two postsynaptic proteins, drebrin (Figure 3B), and PSD95 (Figure $3 C$ ); and three presynaptic proteins, synaptophysin (Figure 3D), syntaxin 1 (Figure 3E), and SNAP25 (Figure 3 F). As an initial control, we measured PSD95 and synaptophysin levels in neuronal cultures treated with LPS for $72 \mathrm{~h}$ in the absence of microglia, and confirmed no effect on these synaptic proteins (\% PSD95 levels: $100 \pm 9.2$ and $94.5 \pm 8.6$, without and with LPS respectively; \% synaptophysin levels: $100 \pm 10.6$ and $95.9 \pm 1.6$, without and with LPS respectively). However, when LPS was added to the WT microglia/neuron co-culture (Figure 3), there was a significant decrease in three of the five synaptic proteins measured: namely, drebrin, synaptophysin, and SNAP25. Microglia p38 $\alpha$ is involved in these responses, as demonstrated by the observation that the absence of microglia $38 \alpha$ protected against the LPS-induced decrease in drebrin, synaptophysin and SNAP25. There were no significant LPS-induced changes in levels of PSD95 or syntaxin 1 in co-cultures with either WT microglia or $\mathrm{p} 38 \alpha \mathrm{KO}$ microglia.

\section{Microglial p38 $\alpha$ MAPK-dependent TNF $\alpha$ is involved in LPS-induced neuronal death}

Activated microglia can produce a variety of secreted molecules that have the potential to be neurotoxic, including proinflammatory cytokines such as TNF $\alpha$. We have previously reported [23] that the production of TNF $\alpha$ from activated microglia is dependent on the p38 $\alpha$ MAPK pathway. Therefore, this cytokine was a logical candidate to test for involvement in the microglia-induced neurotoxicity seen in our co-cultures. We first determined if LPS-treated microglia/neuron co-cultures are associated with elevated TNF $\alpha$ level. In neurons cultured alone, with or without LPS, TNF $\alpha$ was below the limit of detection $(<3.4 \mathrm{pg} / \mathrm{ml})$. In WT microglia/neuron co-cultures, LPS stimulated a $\sim 6$-fold increase in TNF $\alpha$ levels (Figure 4A). The levels of TNF $\alpha$ reached maximum after $24 \mathrm{~h}$ of LPS treatment and remained high until the $72 \mathrm{~h}$ time-point (data for $48 \mathrm{~h}$ not shown). In co-cultures with $\mathrm{p} 38 \alpha \mathrm{KO}$ microglia stimulated with LPS, the levels of TNF $\alpha$ were significantly $(\mathrm{p}<0.0005)$ less than in co-cultures with WT microglia at all three time-points.

We next addressed the question of whether TNF $\alpha$ overproduction is essential for the neurotoxicity observed in the microglia/neuron co-cultures. To test this hypothesis, we used a TNF $\alpha$ neutralizing antibody to decrease the TNF $\alpha$ levels in WT microglia/neuron co-culture. As shown in Figure 4B, LPS caused $40 \%$ neuronal death. When a TNF $\alpha$ neutralizing antibody was added to the culture, we found a concentrationdependent neuroprotection. At a concentration of 50 $\mathrm{ng} / \mathrm{ml}$ or higher of the neutralizing antibody there was a significant reduction in LPS-induced neuronal death, reaching $100 \%$ neuronal survival at $5 \mu \mathrm{g} / \mathrm{ml}$ anti-TNF $\alpha$ antibody. In contrast, the administration of non-immune isotype control antibody $(5 \mu \mathrm{g} / \mathrm{ml})$ failed to protect neurons from the LPS-induced neuronal death (Figure 4B). 


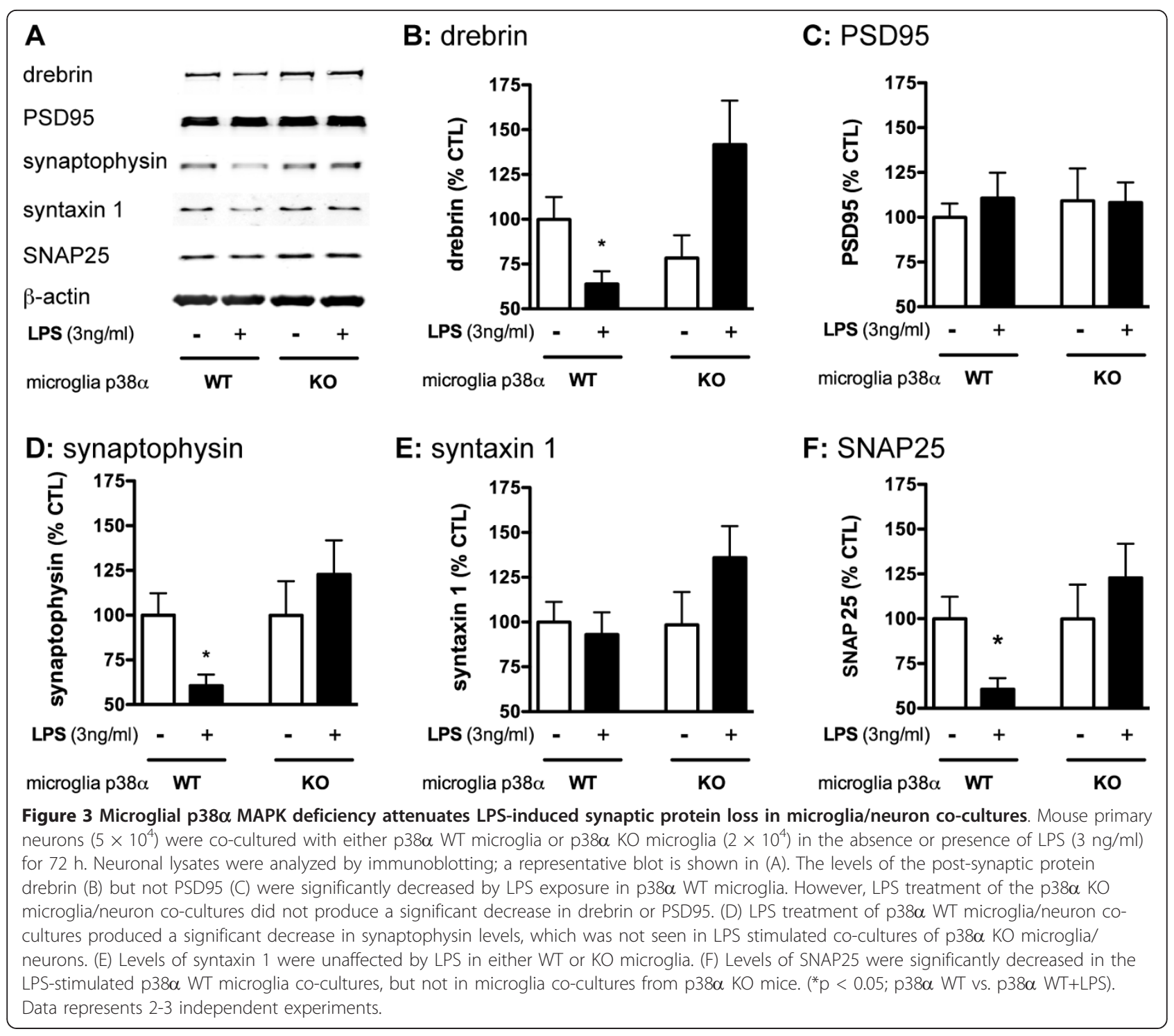

These results suggest that blocking TNF $\alpha$ in WT microglia/neuron co-cultures is sufficient to prevent LPSinduced neuronal death.

As a complementary approach to determining the involvement of TNF $\alpha$ in the LPS-induced neurotoxicity, we tested whether the enhanced neuronal survival seen in p $38 \alpha \mathrm{KO}$ microglia/neuron co-cultures could be influenced by adding back TNF $\alpha$ to levels seen in WT microglia/neuron co-cultures. As shown in Figure 4A, in the $\mathrm{p} 38 \alpha \mathrm{KO}$ microglia/neuron co-cultures treated with LPS, TNF $\alpha$ is decreased on average $\sim 5 \mathrm{ng} / \mathrm{ml}$ compared to WT. Therefore, two concentrations of TNF $\alpha$ ( 5 and $10 \mathrm{ng} / \mathrm{ml}$ ) were administered along with LPS to the p38 $\alpha$ KO microglia/neuron co-cultures, and neuronal survival was measured after treatment for $72 \mathrm{~h}$. At a concentration of $5 \mathrm{ng} / \mathrm{ml}$ or $10 \mathrm{ng} / \mathrm{ml} \mathrm{TNF} \alpha$, we found a significant concentration-dependent increase in neuronal death compared to the $\mathrm{p} 38 \alpha \mathrm{KO}$ microglia/ neuron co-cultures that were stimulated with LPS alone (Figure 4C). These results demonstrate that addition of TNF $\alpha$ to $\mathrm{p} 38 \alpha \mathrm{KO}$ microglia/neuron co-cultures increases LPS-induced neurotoxicity to levels comparable to that seen in WT microglia/neuron co-cultures.

\section{Microglial p38 $\alpha$ MAPK-dependent TNF $\alpha$ is involved in LPS-induced neurite degeneration}

Following LPS stimulation of microglia/neuron co-cultures we found, by immunocytochemistry for MAP-2, that neurites of surviving neurons had marked swellings, with an appearance of beads on a string (see arrow, Figure 5A). These swellings, or blebs, were not seen in cocultures without LPS stimulation (see arrowhead, Figure $5 \mathrm{~A}$ ). In order to quantify these observations, we used Sholl analysis [27] to quantify the total number of 


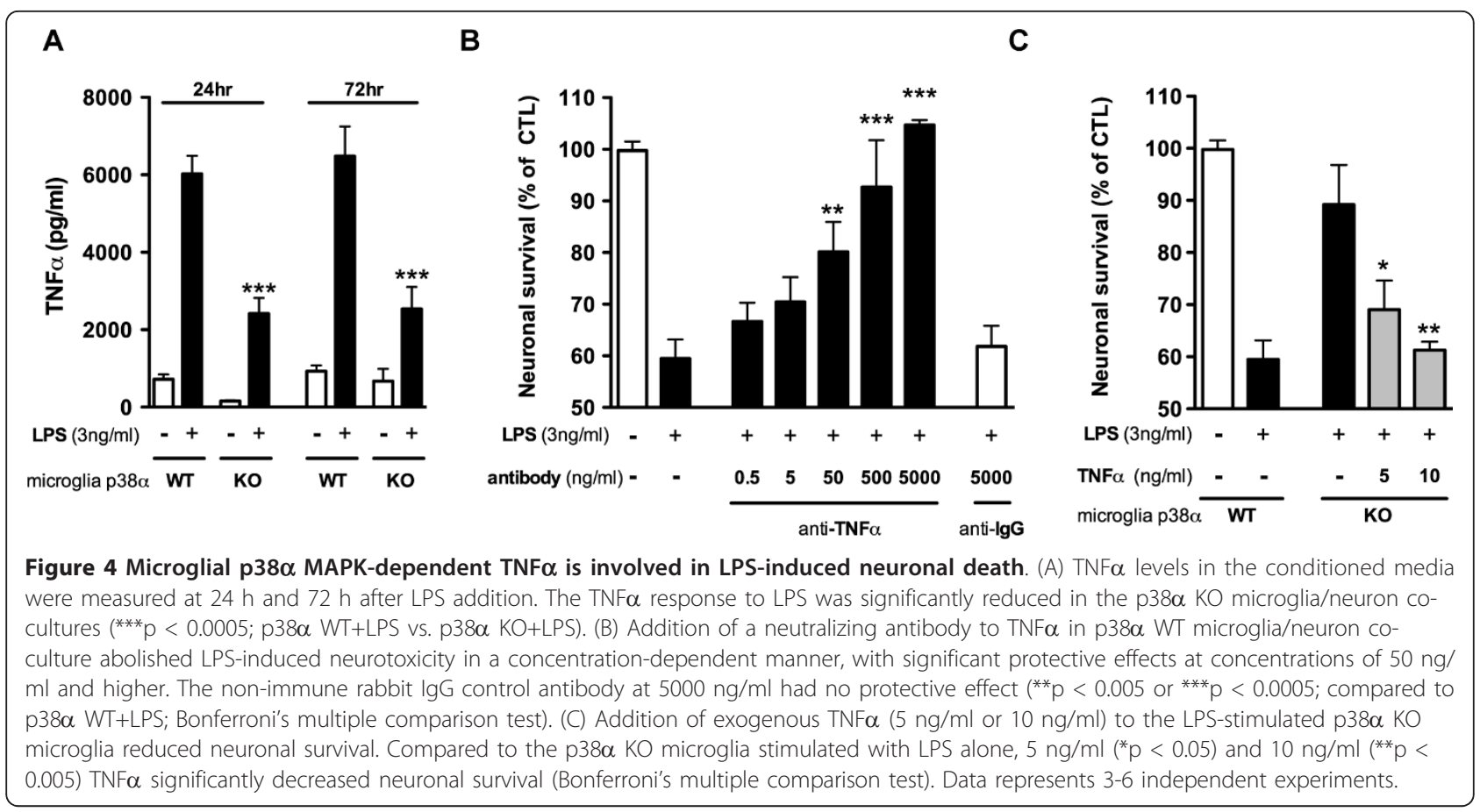

intersections that neurites made with the concentric circles (Figure 5B). We found no significant difference between the groups in terms of the total number of neurite intersections, irrespective of whether the neurite was smooth or had blebs $(28.9 \pm 2.74$ average across groups). However, as shown in Figure 5C, when we quantified only the neurites that are smooth, indicative of a 'healthy' neurite, we found that LPS-stimulated WT microglia/neuron co-cultures showed a highly significant decrease in the healthy neurite arborization compared to the non-LPS-stimulated co-culture. Moreover, the degeneration of the neurites was dependent on p $38 \alpha$ MAPK produced TNF $\alpha$. This was demonstrated by a significant recovery in the numbers of healthy neurites either by addition of a TNF $\alpha$ blocking antibody to WT microglia or by $\mathrm{p} 38 \alpha$ MAPK deficiency in microglia. We further found that adding back TNF $\alpha$ to the p38 $\alpha \mathrm{KO}$ microglia/neuron co-cultures recapitulated the neurodegenerative phenotype seen with the LPS-stimulated WT microglia.

\section{Discussion}

In the current study, we used microglia/neuron co-cultures to document several important findings about the mechanisms by which activated microglia can produce neurodegenerative responses. First, the importance of microglia p38 $\alpha$ MAPK signaling was demonstrated by the observations that neurons in co-culture with p $38 \alpha-$ deficient microglia were protected against LPS-induced neurotoxicity, synaptic protein loss, and neurite degeneration. Second, p38 $\alpha$-dependent microglia TNF $\alpha$ production was shown to be involved in the mechanism of the LPS-induced neuron damage by the findings that p38 $\alpha$ KO microglia produce much less TNF $\alpha$ in response to LPS compared to WT microglia, that adding back TNF $\alpha$ to p38 $\alpha$ KO microglia increases the LPSinduced neurotoxicity, and that neutralization of TNF $\alpha$ in WT microglia decreases the LPS-induced neuron damage. Altogether, our results demonstrate the critical importance of the $\mathrm{p} 38 \alpha$ MAPK signaling pathway and overproduction of the proinflammatory cytokine TNF $\alpha$ in the dysregulated microglia inflammatory responses to an LPS stressor, leading to microglia-induced neuronal dysfunction.

Our demonstration that microglia p38 $\alpha$ MAPK signaling is important in the mechanism of LPS-induced neuron damage is consistent with numerous findings that have implicated p38 MAPK activation in the process of neuronal death in a variety of neurodegenerative disorders. In addition, our studies here using cell-selective, isoform-specific $\mathrm{KO}$ mice extend previous findings by showing that the p38 $\alpha$ MAPK isoform in microglia is a key mediator of LPS-induced neuronal and synaptic dysfunction. We also provide evidence that one mechanism by which LPS activation of microglia p38 $\alpha$ MAPK signaling leads to neuron death is through up-regulation of the proinflammatory cytokine TNF $\alpha$.

The p38 MAPK family consists of four major isoforms (p38 $\alpha, \beta, \delta, \gamma$ ) that have different cell and tissue expression patterns, substrate specificities, and functions [for 


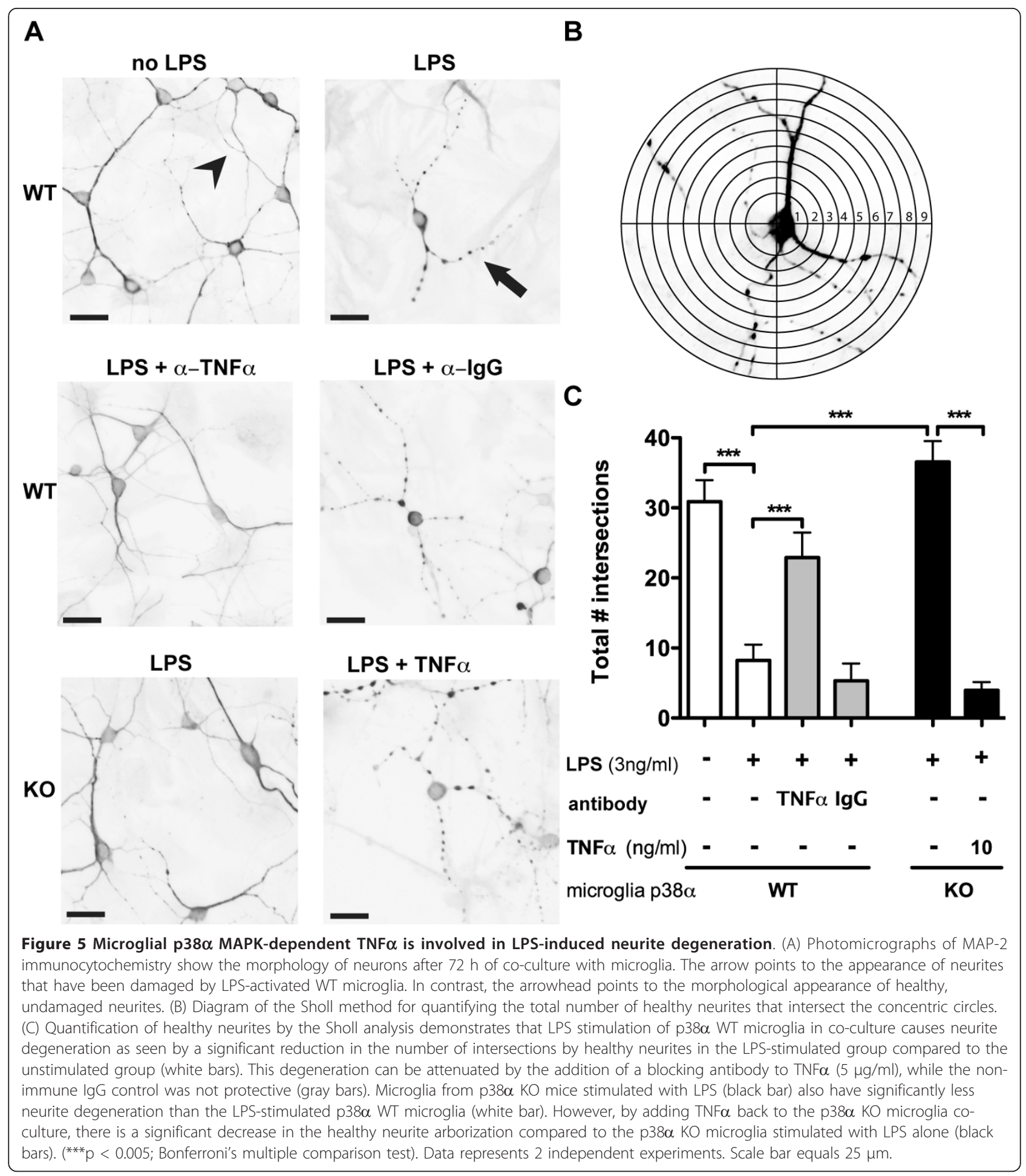

reviews, see: $[14,28]]$. The patterns of expression and activation of the $\mathrm{p} 38 \alpha$ isoform in peripheral immune cells $[29,30]$ suggested that this isoform might play a major role in the inflammatory response. Early attempts using genetic $\mathrm{KO}$ approaches to explore the role of p38 $\alpha$ in inflammatory responses were hampered because of embryonic lethality seen with global KO of p38 $\alpha$. However, a number of more recent studies have used conditional ablation of $\mathrm{p} 38 \alpha$ in specific cell types to provide direct evidence that the p38 $\alpha$ isoform is of central importance for many peripheral inflammatory responses, such as inflammation-induced arthritic bone 
loss [31], inflammatory skin injuries [13], inflammatory responses of myeloid cells in an experimental colitis model [32], immune cell recruitment and pathogen clearance in intestinal epithelial cells [33], and LPSinduced cytokine production in macrophages [25]. These and other studies using selective p38 $\alpha$ inhibitors and drug-resistant forms of the kinase have demonstrated the importance of $\mathrm{p} 38 \alpha$ signaling in mediating peripheral inflammatory responses [34-37].

Although there is broad agreement that $\mathrm{p} 38 \alpha$ plays a key role in cytokine production and other inflammatory responses in peripheral immune cells, the contribution of p38 $\alpha$ to pathological microglial activation and detrimental inflammation in CNS disorders is less well understood. Increasing evidence suggests that p38 signaling cascades contribute to CNS cytokine overproduction and neurodegenerative sequelae [for reviews, see: $[14,38,39]]$, but few studies have tested the specific role of microglia p38 $\alpha$. Expression of the p38 $\alpha$ isoform in microglia was reported to increase early after transient global ischemia [40], and administration of p38 inhibitors reduced infarct volume $[15,41]$ and suppressed proinflammatory cytokine production [41]. We recently demonstrated [23] a direct linkage between microglia p38 $\alpha$ and proinflammatory cytokine production in response to different stressors by showing that inhibition of p38 $\alpha$ in microglia with either a pharmacologic or genetic approach suppresses proinflammatory cytokine up-regulation induced by toll-like receptor ligands or beta-amyloid.

In the present study, we explored the consequences of the microglial p38 $\alpha$-dependent proinflammatory cytokine response on neuronal endpoints. By using microglia deficient in p38 $\alpha$, we showed definitively that microglial p38 $\alpha$ is critical for LPS-induced neuron dysfunction and we implicated p38 $\alpha$-dependent production of the proinflammatory cytokine TNF $\alpha$ in the mechanism of neuron damage. The potential involvement of TNF $\alpha$ was not unexpected, as this proinflammatory cytokine has been shown to induce neurotoxicity in models of CNS neurodegenerative disorders [42-44], and blocking TNF $\alpha$ signaling can be neuroprotective $[45,46]$. However, TNF $\alpha$ is pleiotropic and can also have neuroprotective functions [for review, see: [47]]. Multiple factors influence whether TNF $\alpha$ will exert neurotoxic or neuroprotective actions, including the level and duration of expression in a particular cell type or brain region, the microglia activation state, the particular disease or disease stage, the levels of different TNF receptors and adapter proteins, and the upstream activators and downstream effectors in the signaling pathways. Thus, it was somewhat surprising that microglia $\mathrm{p} 38 \alpha$-dependent production of TNFa in response to an LPS insult appeared to be sufficient to induce neuron death, as evidenced by the observations that anti-TNF $\alpha$ antibody treatment resulted in increased neuronal survival back to control values, and addition of TNF $\alpha$ to $\mathrm{KO}$ microglia reduced neuronal survival to the same levels as WT. Altogether, our data demonstrate that microglia p38 $\alpha$ activation in response to an LPS stressor stimulus and the consequent dysregulated TNF $\alpha$ signaling can lead to neuron damage.

Of note is our finding that $\mathrm{p} 38 \alpha$ MAPK deficiency in microglia attenuates LPS-induced loss of specific synaptic proteins in the co-cultures. Previous studies have shown a correlation between p38 MAPK activation and a decline in synaptophysin levels in AD transgenic mouse models and in primary microglia and cortical neuron co-cultures stimulated with LPS $[48,49]$, and pharmacological inhibition of p38 $\alpha$ MAPK significantly reduced TNF $\alpha$ and IL-1 $\beta$ production and prevented synaptophysin loss in an AD mouse model [24]. Our results here demonstrate for the first time a linkage of p38 $\alpha$ MAPK and TNF $\alpha$ to LPS-induced decreases in SNAP25 and drebrin. Because drebrin, a postsynaptic protein found within dendritic spines, is important for spine morphogenesis and maintenance [50,51], future studies should examine in more detail the mechanisms by which p38 $\alpha$ MAPK influences dendritic pathology and synaptic deterioration such as seen in many neurodegenerative disorders. Future studies should also explore whether microglia p38 $\alpha$ MAPK is involved in beneficial responses of activated microglia, as the current study focused only on detrimental consequences of microglia p38 $\alpha$ activation.

\section{Conclusions}

We report that p38 $\alpha$ MAPK in microglia plays a critical role in activated microglia-mediated neurotoxicity, loss of synaptic proteins, and neurite degeneration via a mechanism involving TNF $\alpha$ signaling. These results suggest that selective targeting of the p38 $\alpha$ MAPK signaling pathway should be explored as a potential therapeutic strategy for the treatment of CNS disorders where overproduction of proinflammatory cytokines is implicated in disease progression.

\section{Methods}

\section{Animals}

All experiments were conducted in accordance with the principles of animal care and experimentation in the Guide For the Care and Use of Laboratory Animals. The Institutional Animal Care and Use Committee of the University of Kentucky approved the use of animals in this study. C57BL/6 mice were obtained from Harlan Laboratories. The p38 $\alpha$ MAPK conditional knockout mice were generated as previously described $[23,25]$, following a standard breeding scheme for conditional gene 
inactivation [52]. The first exon of the p38 $\alpha$ gene (MAPK14) was flanked by two loxP sites. The mice were backcrossed to homozygosity so that both alleles of the $\mathrm{p} 38 \alpha$ gene contained loxP sites $\left(\mathrm{p} 38 \alpha^{\mathrm{fl} / f \mathrm{fl}}\right)$ and maintained on a C57BL/6 background. LysM-Cre mice expressing the Cre recombinase transgene under control of the lysozyme M promoter (B6.129-Lyzs ${ }^{\mathrm{tm} 1(\mathrm{cre}) \mathrm{Ifo} / \mathrm{J})}$ were then crossed with the $\mathrm{p} 38 \alpha^{\mathrm{fl} / \mathrm{fl}}$ mice. The LysMCre ${ }^{+} \mathrm{p} 38 \alpha^{\mathrm{fl} / \mathrm{fl}}$ offspring were then crossed with the $\mathrm{p} 38 \alpha^{\mathrm{fl} / \mathrm{fl}}$ mice to generate experimental and control animals. This generates litters where $\sim 50 \%$ mice are $\mathrm{p} 38 \mathrm{\alpha}^{\mathrm{fl} / \mathrm{fl}(+\mathrm{cre})}(\mathrm{KO})$ and $\sim 50 \%$ are $\mathrm{p} 38 \alpha^{\mathrm{fl} / \mathrm{fl}(-\mathrm{cre})}$ (used as WT controls). The restricted cell-type expression of the lysozyme promoter $[53,54]$ results in cell-specific deletion of p38 $\alpha$ MAPK in myeloid cells including microglia. Genotyping was performed by Transnetyx, Inc (Cordova, TN).

\section{Primary neuronal culture}

Primary neuronal cultures were derived from embryonic day 18, C57BL/6 mice, as previously described [26]. Briefly, cerebral cortices were dissected and the meninges were removed. Cells were dissociated by trypsinization (0.25\% trypsin, $2.21 \mathrm{mM}$ EDTA) for $15 \mathrm{~min}$ at $37^{\circ} \mathrm{C}$ and triturated, followed by passing through a 70 $\mu \mathrm{m}$ nylon mesh cell strainer. The neurons were plated onto poly-D-lysine-coated 12 -mm glass coverslips at a density of $5 \times 10^{4} /$ well in 24 well plates. Neurons were grown in neurobasal medium (Invitrogen) containing $2 \%$ B27 supplement (Invitrogen), $0.5 \mathrm{mM} \mathrm{L}$-glutamine, (Mediatech), and $100 \mathrm{IU} / \mathrm{ml}$ penicillin, $100 \mu \mathrm{g} / \mathrm{ml}$ streptomycin (Mediatech); no serum or mitosis inhibitors were used. Every 3 days, $50 \%$ of the media was replenished with fresh medium. The purity of the primary neuronal cultures was verified as $93 \%$ by immunocytochemistry for the neuronal marker NeuN, astrocyte marker GFAP, and microglia marker Iba-1 (data not shown).

\section{Microglia culture}

Microglia cultures were prepared as previously described [23]. Briefly, mixed glial cultures ( $\sim 95 \%$ astrocytes, $\sim 5 \%$ microglia) were prepared from the cerebral cortices of 1-3 day old mice. The tissue was trypsinized as above, and the cells were resuspended in glia complete medium $[\alpha$-minimum essential medium ( $\alpha$-MEM; Mediatech) supplemented with $10 \%$ fetal bovine serum (FBS) (US Characterized FBS; Hyclone; Cat no. SH30071.03), 100 $\mathrm{IU} / \mathrm{ml}$ penicillin, $100 \mu \mathrm{g} / \mathrm{ml}$ streptomycin (Mediatech) and $2 \mathrm{mM}$ L-Glutamine (Mediatech)]. After 10-14 days in culture, microglia were isolated from the mixed glial cultures by the shake-off procedure [55]. Specifically, loosely attached microglia were shaken off in an incubator shaker at $250 \mathrm{rpm}$ for $2 \mathrm{~h}$ at $37^{\circ} \mathrm{C}$, the cell-containing medium was centrifuged at $1100 \mathrm{rpm}$ for $3 \mathrm{~min}$, and the cells were seeded onto 12 -mm glass coverslip at the density of $2 \times 10^{4}$ in 24 well plates, unless otherwise specified. Prior to plating the microglia on the coverslip, three equally spaced $1 \mathrm{~mm}$ glass beads (Borosilicate; Sigma) were attached to the coverslip with paraffin wax. The microglia cultures were verified to be $>99 \%$ microglia by immunocytochemistry. Microglia were incubated for one day before placing into co-culture with neurons.

\section{Primary neuron/microglia co-culture and cell treatments}

Following previously described methods [26], after 7-9 days in culture, neurons on coverslips were co-cultured with mouse microglia by placing the microglia-containing coverslips cell side down into the neuron-containing wells. In this co-culture system, the microglia and neurons are in close apposition and share the same neurobasal/B27 culture media, but are separated by the $1 \mathrm{~mm}$ glass beads and do not have direct cell-cell contact. Lipopolysaccharide (LPS) from Salmonella typhimurium (Sigma) was resuspended in sterile saline at $100 \mathrm{mg} / \mathrm{ml}$, and was used at a final concentration of $3 \mathrm{ng} / \mathrm{ml}$ for all experiments. A rat monoclonal $\mathrm{IgG}_{1}$, anti-mouse TNF $\alpha$ neutralization antibody (clone \# MP6-XT22) with a reported $50 \%$ neutralization dose in the range of 0.15 $0.75 \mu \mathrm{g} / \mathrm{ml}$, was reconstituted in sterile PBS according to manufacturer specifications (R\&D Systems). A rat $I G_{1}$ monoclonal antibody (clone \# 43414) was used as a non-immune isotype control antibody (R\&D Systems). Treatment with either antibody occurred $1 \mathrm{~h}$ prior to LPS treatment. Recombinant mouse TNF $\alpha$ (aa 80-235; R\&D systems) was added at the same time as the LPS treatment.

\section{Neuronal Viability Assay}

Neuron viability was assayed by trypan blue exclusion [26]. Neuron-containing coverslips were incubated with $0.2 \%$ trypan blue in Hanks' Balanced Salt Solution (HBSS) for $2 \mathrm{~min}$ in $37^{\circ} \mathrm{C}$ incubator and then rinsed 3 times with HBSS. Neurons were viewed under brightfield microscopy at $200 \times$ final magnification. Three to five fields were chosen per coverslip, and a total of 150 to 560 cells were counted per coverslip. Trypan bluepositive and negative neurons were counted per field and the ratio of negative cells to the total cells was taken as the index of neuronal survival rate.

\section{Immunocytochemistry}

Cells were fixed with $3.7 \%$ formalin containing $0.1 \%$ Triton $\mathrm{X}-100$ in PBS for $10 \mathrm{~min}$ at room temperature. After washing three times with PBS, the coverslips were incubated with blocking buffer (PBS containing 5\% goat serum, 3\% bovine serum albumin (BSA; Fisher Scientific), $0.1 \%$ Triton $\mathrm{X}-100$ ) for $30 \mathrm{~min}$ at room temperature. Primary antibodies were diluted in blocking buffer 
and incubated with the cells at room temperature for 2 h. Primary antibodies used in this study were: chicken anti-MAP-2 antibody (1:100, Neuromics); mouse antiNeuN (1:100, Millipore); rat anti-GFAP (1:1000, Invitrogen); rabbit anti-IBA1 (1:1000, Wako); rat anti-CD11b (1:100, Serotec); rat anti-F4/80 (1:100, Serotec); and p38 $\alpha$ (1:100, R\&D Systems). For detection of primary antibodies, species-appropriate Alexa Fluor ${ }^{\circledR}$ fluorescent conjugated secondary antibodies (1:1000, Invitrogen) were incubated in blocking buffer at room temperature for $2 \mathrm{~h}$. Wide field fluorescent photomicrographs were obtained using a Zeiss Axioplan 2 microscope with an Axiocam MRc5 digital camera (Carl Zeiss).

\section{Western blotting and ELISA assays}

Western blotting was performed as previously described [55]. Briefly, whole cell lysates were prepared in sodium dodecyl sulfate (SDS)- containing sample buffer, and equal volumes of lysates were separated by $10.5-14 \%$ SDS-PAGE Criterion precast gel (Bio-Rad Laboratories). Proteins were transferred to nitrocellulose membrane using a dry blotting system (iBlot ${ }^{\circledR}$ Invitrogen). Blots were probed using reagents and manufacturer recommendations for Odyssey Infrared Imaging system (LICOR Biosciences), with the following primary antibodies: mouse anti-drebrin (1:5000, Abcam); rabbit antiPSD95 (1:2000, Cell Signaling); mouse anti-synaptophysin (1:1000, Millipore); rabbit anti-syntaxin 1 (1:10, 000, Millipore), mouse anti-SNAP 25 (1:4000, BD Biosciences); rabbit anti-p38 $\alpha / \beta$ (1:1000, Cell Signaling), and mouse anti- $\beta$-Actin (1:10, 000, Cell Signaling). Blots were visualized and analyzed on the Odyssey Infrared imaging system (LI-COR Biosciences), and integrated intensity values were used in statistics.

After $24 \mathrm{~h}, 48 \mathrm{~h}$, and $72 \mathrm{~h}$ in the co-cultures, $20 \mu \mathrm{l}$ conditioned medium was harvested for TNF $\alpha$ ELISA assay using kits from Meso Scale Discovery (MSD) according to the manufacturer's instructions.

\section{Sholl analysis}

The Sholl method [27] was used in the quantification of MAP-2 labeled neurites. A series of concentric circles were drawn at $10 \mu \mathrm{m}$ intervals starting with a diameter of $20 \mu \mathrm{m}$ to a final diameter of $200 \mu \mathrm{m}$. Intersections of smooth or blebbed neurites with the concentric circles were counted. The total number of intersections for each neuron was plotted as a measure of neurite arborization. Per experimental condition, 20-30 neurons were analyzed from two independent experiments by an observer blinded to treatment conditions.

\section{Statistics}

Statistical analysis was conducted using GraphPad prism software V.5 (GraphPad Software, La Jolla, CA). Unless otherwise indicated, values are expressed as mean \pm SEM. Groups of two were compared by unpaired $t$-Test. One-way ANOVA followed by Bonferroni's multiple comparison test was used for comparisons among three or more groups. Statistical significance was defined as $\mathrm{p}$ $<0.05$.

\section{List of abbreviations}

(AD): Alzheimer's disease; (KO): knockout; (WT): wild-type; (LPS): lipopolysaccharide; (MAPK): mitogen-activated protein kinase; (TNF): tumor necrosis factor

\section{Acknowledgements}

This work was supported in part by Alzheimer's Association Zenith grant ZEN-09-134506 and NIH grant R01 NS064247 to LVE. ADB is supported by $\mathrm{NIH}$ fellowship F32 AG037280. We thank Dr. Huiping Jiang at Boehringer Ingelheim Pharmaceuticals, Inc. and Dr. Jiahuai Han at The Scripps Research Institute for the kind gifts of the knockout mice.

\section{Author details}

'Sanders-Brown Center on Aging, University of Kentucky, Lexington, KY 40536 USA. ${ }^{2}$ Department of Anatomy and Neurobiology, University of Kentucky, Lexington, KY 40536 USA.

\section{Authors' contributions}

$B X, A D B$ and LVE designed the studies. BX performed the experiments in cell culture. BX and ADB performed the data analysis. BX and ADB jointly drafted the manuscript together with LVE. All authors read and approved the final version. BX and $A D B$ contributed equally to this study.

\section{Competing interests}

The authors declare that they have no competing interests.

Received: 30 September 2011 Accepted: 20 December 2011 Published: 20 December 2011

\section{References}

1. Van Eldik LJ, Thompson WL, Ralay Ranaivo H, Behanna HA, Martin Watterson D: Glia proinflammatory cytokine upregulation as a therapeutic target for neurodegenerative diseases: function-based and target-based discovery approaches. Int Rev Neurobiol 2007, 82:277-296.

2. Lloyd E, Somera-Molina K, Van Eldik LJ, Watterson DM, Wainwright MS: Suppression of acute proinflammatory cytokine and chemokine upregulation by post-injury administration of a novel small molecule improves long-term neurologic outcome in a mouse model of traumatic brain injury. J Neuroinflammation 2008, 5:28.

3. Morganti-Kossmann MC, Satgunaseelan L, Bye N, Kossmann T: Modulation of immune response by head injury. Injury 2007, 38(12):1392-1400.

4. Keane RW, Davis AR, Dietrich WD: Inflammatory and apoptotic signaling after spinal cord injury. J Neurotrauma 2006, 23(3-4):335-344.

5. Nilupul Perera M, Ma HK, Arakawa S, Howells DW, Markus R, Rowe CC, Donnan GA: Inflammation following stroke. J Clin Neurosci 2006, 13(1):1-8.

6. Ransohoff RM, Perry $\mathrm{VH}$ : Microglial physiology: unique stimuli, specialized responses. Annu Rev Immunol 2009, 27:119-145.

7. Kettenmann H, Hanisch UK, Noda M, Verkhratsky A: Physiology of microglia. Physiol Rev 2011, 91(2):461-553.

8. Nimmerjahn A, Kirchhoff F, Helmchen F: Resting microglial cells are highly dynamic surveillants of brain parenchyma in vivo. Science 2005, 308(5726):1314-1318

9. Davalos D, Grutzendler J, Yang G, Kim JV, Zuo Y, Jung S, Littman DR, Dustin ML, Gan WB: ATP mediates rapid microglial response to local brain injury in vivo. Nat Neurosci 2005, 8(6):752-758.

10. Mallat M, Marin-Teva JL, Cheret C: Phagocytosis in the developing CNS: more than clearing the corpses. Curr Opin Neurobiol 2005, 15(1):101-107.

11. Kaminska B: MAPK signalling pathways as molecular targets for antiinflammatory therapy-from molecular mechanisms to therapeutic benefits. Biochim Biophys Acta 2005, 1754(1-2):253-262. 
12. Saklatvala J: The p38 MAP kinase pathway as a therapeutic target in inflammatory disease. Curr Opin Pharmacol 2004, 4(4):372-377.

13. Kim C, Sano Y, Todorova K, Carlson BA, Arpa L, Celada A, Lawrence T, Otsu K, Brissette JL, Arthur JS, Park JM: The kinase p38 alpha serves cell type-specific inflammatory functions in skin injury and coordinates proand anti-inflammatory gene expression. Nat Immunol 2008 , 9(9):1019-1027.

14. Bachstetter AD, Eldik LJV: The p38 MAP Kinase Family as Regulators of Proinflammatory Cytokine Production in Degenerative Diseases of the CNS. Aging and Disease 2010, 1(3):199-211.

15. Barone FC, Irving EA, Ray AM, Lee JC, Kassis S, Kumar S, Badger AM, White RF, McVey MJ, Legos JJ, Erhardt JA, Nelson AH, Ohlstein EH, Hunter AJ, Ward K, Smith BR, Adams JL, Parsons AA: SB 239063, a secondgeneration p38 mitogen-activated protein kinase inhibitor, reduces brain injury and neurological deficits in cerebral focal ischemia. $J$ Pharmacol Exp Ther 2001, 296(2):312-321.

16. Legos JJ, Erhardt JA, White RF, Lenhard SC, Chandra S, Parsons AA, Tuma RF, Barone FC: SB 239063, a novel p38 inhibitor, attenuates early neuronal injury following ischemia. Brain Res 2001, 892(1):70-77.

17. Onyango IG, Tuttle JB, Bennett JP Jr: Activation of p38 and Nacetylcysteine-sensitive c-Jun $\mathrm{NH} 2$-terminal kinase signaling cascades is required for induction of apoptosis in Parkinson's disease cybrids. Mol Cell Neurosci 2005, 28(3):452-461.

18. Wilms H, Rosenstiel P, Sievers J, Deuschl G, Zecca L, Lucius R: Activation of microglia by human neuromelanin is NF-kappaB dependent and involves p38 mitogen-activated protein kinase: implications for Parkinson's disease. Faseb J 2003, 17(3):500-502

19. Xing $B$, Xin T, Hunter RL, Bing G: Pioglitazone inhibition of lipopolysaccharide-induced nitric oxide synthase is associated with altered activity of p38 MAP kinase and PI3K/Akt. J Neuroinflammation 2008, 5:4.

20. Sun A, Liu M, Nguyen XV, Bing G: P38 MAP kinase is activated at early stages in Alzheimer's disease brain. Exp Neurol 2003, 183(2):394-405.

21. Hensley K, Floyd RA, Zheng NY, Nael R, Robinson KA, Nguyen X, Pye QN, Stewart CA, Geddes J, Markesbery WR, Patel E, Johnson GV, Bing G: p38 kinase is activated in the Alzheimer's disease brain. J Neurochem 1999, 72(5):2053-2058

22. Pei JJ, Braak E, Braak H, Grundke-lqbal I, labal K, Winblad B, Cowburn RF: Localization of active forms of C-jun kinase (JNK) and p38 kinase in Alzheimer's disease brains at different stages of neurofibrillary degeneration. J Alzheimers Dis 2001, 3(1):41-48.

23. Bachstetter AD, Xing $B$, de Almeida $L$, Dimayuga ER, Watterson DM, Van Eldik $L$ : Microglial p38alpha MAPK is a key regulator of proinflammatory cytokine up-regulation induced by toll-like receptor (TLR) ligands or beta-amyloid (Abeta). J Neuroinflammation 2011, 8(1):79.

24. Munoz L, Ralay Ranaivo H, Roy SM, Hu W, Craft JM, McNamara LK, Chico LW, Van Eldik L, Watterson DM: A novel p38 alpha MAPK inhibitor suppresses brain proinflammatory cytokine up-regulation and attenuates synaptic dysfunction and behavioral deficits in an Alzheimer's disease mouse model. J Neuroinflammation 2007, 4:21.

25. Kang YJ, Chen J, Otsuka M, Mols J, Ren S, Wang Y, Han J: Macrophage deletion of p38alpha partially impairs lipopolysaccharide-induced cellular activation. J Immunol 2008, 180(7):5075-5082.

26. Xie Z, Smith CJ, Van Eldik LJ: Activated glia induce neuron death via MAP kinase signaling pathways involving JNK and p38. Glia 2004, 45(2):170-179.

27. Sholl DA: Dendritic organization in the neurons of the visual and motor cortices of the cat. J Anat 1953, 87(4):387-406

28. Cuenda A, Rousseau S: p38 MAP-kinases pathway regulation, function and role in human diseases. Biochim Biophys Acta 2007, 1773(8):1358-1375.

29. Hale KK, Trollinger D, Rihanek M, Manthey CL: Differential expression and activation of p38 mitogen-activated protein kinase alpha, beta, gamma, and delta in inflammatory cell lineages. J Immunol 1999, 162(7):4246-4252.

30. Schieven GL: The biology of p38 kinase: a central role in inflammation. Curr Top Med Chem 2005, 5(10):921-928.
31. Bohm C, Hayer S, Kilian A, Zaiss MM, Finger S, Hess A, Engelke K, Kollias G, Kronke G, Zwerina J, Schett G, David JP: The alpha-isoform of p38 MAPK specifically regulates arthritic bone loss. J Immunol 2009, 183(9):5938-5947.

32. Otsuka M, Kang YJ, Ren J, Jiang H, Wang Y, Omata M, Han J: Distinct effects of p38alpha deletion in myeloid lineage and gut epithelia in mouse models of inflammatory bowel disease. Gastroenterology 2010, 138(4):1255-1265, 1265 e1251-1259.

33. Kang YJ, Otsuka M, van den Berg A, Hong L, Huang Z, Wu X, Zhang DW, Vallance BA, Tobias PS, Han J: Epithelial p38alpha controls immune cell recruitment in the colonic mucosa. PLoS Pathog 2010, 6(6):e1000934.

34. O'Keefe SJ, Mudgett JS, Cupo S, Parsons JN, Chartrain NA, Fitzgerald C, Chen SL, Lowitz K, Rasa C, Visco D, Luell S, Carballo-Jane E, Owens K, Zaller DM: Chemical genetics define the roles of p38alpha and p38beta in acute and chronic inflammation. J Biol Chem 2007, 282(48):34663-34671

35. Guo X, Gerl RE, Schrader JW: Defining the involvement of p38alpha MAPK in the production of anti- and proinflammatory cytokines using an SB 203580-resistant form of the kinase. J Biol Chem 2003, 278(25):22237-22242.

36. Schindler JF, Monahan JB, Smith WG: p38 pathway kinases as antiinflammatory drug targets. J Dent Res 2007, 86(9):800-811.

37. Natarajan SR, Doherty JB: P38 MAP kinase inhibitors: evolution of imidazole-based and pyrido-pyrimidin-2-one lead classes. Curr Top Med Chem 2005, 5(10):987-1003.

38. Munoz L, Ammit AJ: Targeting p38 MAPK pathway for the treatment of Alzheimer's disease. Neuropharmacology 2010, 58(3):561-568.

39. Yasuda S, Sugiura H, Tanaka H, Takigami S, Yamagata K: p38 MAP kinase inhibitors as potential therapeutic drugs for neural diseases. Cent Nerv Syst Agents Med Chem 2011, 11(1):45-59.

40. Piao CS, Che Y, Han PL, Lee JK: Delayed and differential induction of p38 MAPK isoforms in microglia and astrocytes in the brain after transient global ischemia. Brain Res Mol Brain Res 2002, 107(2):137-144.

41. Piao CS, Kim JB, Han PL, Lee JK: Administration of the p38 MAPK inhibito SB203580 affords brain protection with a wide therapeutic window against focal ischemic insult. J Neurosci Res 2003, 73(4):537-544.

42. McCoy MK, Tansey MG: TNF signaling inhibition in the CNS: implications for normal brain function and neurodegenerative disease. $J$ Neuroinflammation 2008, 5:45.

43. Montgomery SL, Bowers WJ: Tumor Necrosis Factor-alpha and the Roles it Plays in Homeostatic and Degenerative Processes Within the Central Nervous System. J Neuroimmune Pharmacol 2011.

44. Park KM, Bowers WJ: Tumor necrosis factor-alpha mediated signaling in neuronal homeostasis and dysfunction. Cell Signal 2010, 22(7):977-983.

45. Jeohn GH, Cooper CL, Jang KJ, Liu B, Lee DS, Kim HC, Hong JS: Go6976 inhibits LPS-induced microglial TNFalpha release by suppressing p38 MAP kinase activation. Neuroscience 2002, 114(3):689-697.

46. de Bock F, Derijard B, Dornand J, Bockaert J, Rondouin G: The neuronal death induced by endotoxic shock but not that induced by excitatory amino acids requires TNF-alpha. Eur J Neurosci 1998, 10(10):3107-3114.

47. Sriram K, O'Callaghan JP: Divergent roles for tumor necrosis factor-alpha in the brain. J Neuroimmune Pharmacol 2007, 2(2):140-153.

48. Li Y, Liu L, Barger SW, Griffin WS: Interleukin-1 mediates pathological effects of microglia on tau phosphorylation and on synaptophysin synthesis in cortical neurons through a p38-MAPK pathway. J Neurosci 2003, 23(5):1605-1611.

49. Savage MJ, Lin YG, Ciallella JR, Flood DG, Scott RW: Activation of c-Jun Nterminal kinase and p38 in an Alzheimer's disease model is associated with amyloid deposition. J Neurosci 2002, 22(9):3376-3385.

50. Takahashi H, Mizui T, Shirao T: Down-regulation of drebrin A expression suppresses synaptic targeting of NMDA receptors in developing hippocampal neurones. J Neurochem 2006, 97(Suppl 1):110-115.

51. Majoul I, Shirao T, Sekino Y, Duden R: Many faces of drebrin: from building dendritic spines and stabilizing gap junctions to shaping neurite-like cell processes. Histochem Cell Biol 2007, 127(4):355-361.

52. Kwan KM: Conditional alleles in mice: practical considerations for tissuespecific knockouts. Genesis 2002, 32(2):49-62. 
53. Clarke S, Greaves DR, Chung LP, Tree P, Gordon S: The human lysozyme promoter directs reporter gene expression to activated myelomonocytic cells in transgenic mice. Proc Natl Acad Sci USA 1996, 93(4):1434-1438.

54. Clausen BE, Burkhardt C, Reith W, Renkawitz R, Forster I: Conditional gene targeting in macrophages and granulocytes using LysMcre mice. Transgenic Res 1999, 8(4):265-277.

55. Petrova TV, Akama KT, Van Eldik LJ: Cyclopentenone prostaglandins suppress activation of microglia: down-regulation of inducible nitricoxide synthase by 15-deoxy-Delta12, 14-prostaglandin J2. Proc Natl Acad Sci USA 1999, 96(8):4668-4673.

doi:10.1186/1750-1326-6-84

Cite this article as: Xing et al: Microglial p38 $\alpha$ MAPK is critical for LPS induced neuron degeneration, through a mechanism involving TNF $\alpha$.

Molecular Neurodegeneration 2011 6:84.

\section{Submit your next manuscript to BioMed Central} and take full advantage of:

- Convenient online submission

- Thorough peer review

- No space constraints or color figure charges

- Immediate publication on acceptance

- Inclusion in PubMed, CAS, Scopus and Google Scholar

- Research which is freely available for redistribution

Submit your manuscript at www.biomedcentral.com/submit 